\title{
Narrativa
}

\section{Il canone e l'insegnamento della letteratura italiana oltre frontiera. Risultati di un'inchiesta empirica negli atenei belgi e olandesi}

Natalie Dupré, Monica Jansen, Inge Lanslots e Maria Bonaria Urban

\section{(2) OpenEdition}

1 Journals

Edizione digitale

URL: https://journals.openedition.org/narrativa/803

DOI: $10.4000 /$ narrativa.803

ISSN: 2804-1224

\section{Editore}

Presses universitaires de Paris Nanterre

\section{Edizione cartacea}

Data di pubblicazione: 1 décembre 2016

Paginazione: 55-76

ISBN: 978-2-84016-266-7

ISSN: $1166-3243$

Notizia bibliografica digitale

Natalie Dupré, Monica Jansen, Inge Lanslots e Maria Bonaria Urban, «ll canone e l'insegnamento della letteratura italiana oltre frontiera. Risultati di un'inchiesta empirica negli atenei belgi e olandesi», Narrativa [Online], 38 | 2016, online dal 01 janvier 2022, consultato il 22 février 2022. URL: http:// journals.openedition.org/narrativa/803 ; DOI: https://doi.org/10.4000/narrativa.803

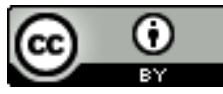

Narrativa est mise à disposition selon les termes de la Licence Creative Commons Attribution 4.0 International. 


\section{Il canone e l'insegnamento della letteratura italiana oltre frontiera. Risultati di un'inchiesta empirica negli atenei belgi e olandesi}

\section{INTRODUZIONE}

Negli ultimi anni si è assistito a una forte ripresa del dibattito attorno al canone letterario. Canonicità, anticanone e processo di costruzione del canone sono concetti tornati a essere di grande interesse anche per quegli studiosi che negli anni precedenti avevano contestato il canone tradizionale invocandone il superamento. Il presente contributo si inserisce in tale dibattito, illustrando i dati di una ricerca empirica condotta nel 2016 presso gli atenei olandesi e belgi, per determinare il processo di selezione e le caratteristiche del canone pedagogico, nonché il suo rapporto con il canone immaginario nell'insegnamento della letteratura italiana all'estero.

Nella prima parte del contributo si fornirà un sintetico quadro teorico dei concetti che sono stati alla base dell'indagine empirica, facendo riferimento sia al dibattito sul canone in Italia sia ad alcune ricerche condotte all'estero che hanno fornito spunti di riflessione e permesso di elaborare gli obiettivi e i riferimenti teorici del questionario. La seconda parte è dedicata alla descrizione sistematica dell'indagine empirica e all'interpretazione dei dati emersi. Nelle conclusioni, infine, si avanza una serie di ipotesi sulla relazione tra il canone in continua formazione nella pratica dell'insegnamento della letteratura italiana all'estero e la teorizzazione del canone della letteratura italiana come strumento di valutazione esclusivo e inclusivo dell'identità culturale nazionale.

\section{IL CANONE E L'INSEGNAMENTO}

DELLA LETTERATURA ITALIANA OLTRE FRONTIERA

\section{DEFINIRE IL CONCETTO DI CANONE}

Oggetto di dispute molto accese negli ultimi anni in cui sono emerse diverse letterature "minoritarie", il concetto di canone sintetizza in realtà diversi signi- 
ficati. Se si parte da un principio "euristico", il canone ha un carattere essenzialmente selettivo e normativo, nel senso che stabilisce quali opere ne fanno parte e implica al contempo l'esclusione automatica di altre, etichettandole come non canoniche. Il criterio di selezione in tale visione si basa su principi universalistici partendo dal presupposto - come fa Italo Calvino nella definizione numero 10 di un "classico" - che "[c]hiamasi classico un libro che si configura come equivalente dell'universo". A tale criterio si appella Carlo Ossola, curatore insieme a Giacomo Jori dell'opera in otto volumi pubblicata dalla UTET nel 2012 in occasione dei 150 anni dell'Italia Unita: la Letteratura italiana. Canone dei classici. Egli stesso così commenta la genesi su Il Sole 24 Ore:

Il canone dei classici attesta questo sguardo all'infinito, nel privilegio che sola ha la letteratura italiana di aver sempre offerto versioni di mondi possibili, configurabili, irraggiungibili: da Dante all'Ariosto, dal Tasso a Galileo, da Leopardi a Pirandello, da Pasolini a Calvino [...]. Questo "canone" obbedisce altresì al memorabile che ogni giorno incontriamo nelle nostre piazze: la nostra civiltà è stata fatta per durare e non per negarsi; ogni secolo è scandito da opere che lo hanno rappresentato nella memoria collettiva: sarebbe possibile pensare alla dignità dell'uomo, anche oggi, senza la Creazione di Adamo, di Michelangelo, nella Sistina? ${ }^{2}$

Matteo di Gesù, in un commento su Nażione indiana a questa grande impresa da lui definita "monumentalizzante", parte invece da un'altra definizione di Calvino di cosa sia un classico, ovvero dalla numero 5: "Un classico è un libro che non ha mai finito di dire quel che ha da dire"3. E aggiunge che il valore dell'opera non è solo intrinseco ma si misura anche dalla sua ricezione che passa inevitabilmente attraverso l'accademia e la critica letteraria ${ }^{4}$. In altre parole, tutto sta nel come ci si posiziona nel confronto con i classici, e qui si potrebbe tornare alla definizione 10 di Calvino che egli stesso commenta come segue per arrivare alla definizione 11: "Ma un classico può stabilire un rapporto altrettanto forte d'opposizione, d'antitesi. [...] Dirò dunque: 11. Il 'tuo' classico è quello che non può esserti indifferente e che ti serve per definire te stesso in rapporto e magari in contrasto con lui" ${ }^{5}$.

1. Calvino, Italo, Perché leggere i classici, Milano, Mondadori, 1991, p. 16.

2. Ossola, Carlo, "La letteratura che ci detta dentro", Il Sole 24 Ore, 9 maggio 2012.

3. Calvino, Italo, Perché leggere i classici, cit., p. 13.

4. Di Gesù, Matteo, "Possiamo sbarazzarci dei classici italiani?", Nazione indiana, 24 novembre 2012, https://www.nazioneindiana.com/2012/11/24/ possiamo-sbarazzarci-dei-classici-italiani/

5. Calvino, Italo, Perché leggere i classici, cit., p. 16. 
All'origine dell'attuale dibattito sul canone c'è, come noto, l'ormai famosa disputa scoppiata all'Università di Stanford nel 1987': il nucleo dello scontro era il concetto stesso di canone e la liceità o meno di aprirlo a testi fino a quel momento considerati non canonici. La messa in discussione del canone classico nasceva dal bisogno sempre più diffuso di rivendicarne l'apertura in direzione di quei campi di ricerca affermatisi con la diffusione dei Cultural Studies (studi postcoloniali, Gender Studies, ecc.). In questo dibattito, Harold Bloom con il suo The Western Canon (1994) incarna la posizione di coloro che difendono la fissità del canone occidentale, affermando la superiorità del principio estetico su quello politico". Come rileva Michela Zompetta, infatti, Bloom ritiene che "l'estetica è un interesse individuale e non sociale, che l'arte è inutile e che è un'illusione pensare che possa diventare una base per l'educazione democratica o il miglioramento sociale"s.

$\mathrm{Al}$ di là delle prese di posizione di detrattori e difensori del canone occidentale, studiosi come John Guillory e, successivamente, Susan V. Gallagher hanno posto l'attenzione su un equivoco alla base di tutta la discussione, cogliendo la differenza sostanziale che esiste fra l'insieme di opere idealmente parti del canone e quelle che effettivamente sono inserite nei programmi d'insegnamento. Esiste infatti un canone ideale che può essere definito "canone immaginario" (imaginary (anon) $)^{9}$ cui si contrappone la selezione di opere effettivamente destinate all'insegnamento, il cosiddetto "canone pedagogico", corrispondente alla lista di autori trattati nei programmi di studi. Mentre il canone immaginario fa riferimento a una totalità di opere, quello pedagogico si basa sul "sillabo" (syllabus), cioè, nelle parole di Guillory, la lista di opere che si leggono in una data classe o sul curriculum, cioè la lista di opere incluse in un programma di studio ${ }^{10}$.

Tale distinzione costituisce il punto di partenza della ricerca empirica qui presentata, il cui duplice obiettivo è l'analisi del processo di selezione del "canone pedagogico" nel contesto universitario olandese e belga, e l'individuazione dei criteri e delle possibili motivazioni alla base delle scelte operate dai

6. Per una panoramica sulle origini del dibattito in ambiente nordamericano si rimanda a ZompetTA, Michela, "Bloom: Un canone occidentale per l'Europa", Sinestesieonline, n. 4, 2013, p. 1, http://www.rivistasinestesie.it/PDF/2013/MAGGIO/6.pdf

7. Bloom, Harold, The Western Canon. The Books and School of the Ages, New York, Harcourt Brace, 1994.

8. ZompetTA, Michela, "Bloom: Un canone occidentale", cit., p. 5.

9. Gallagher, Susan V., "Contingencies and Intersections: The Formation of Pedagogical Canons”, Pedagogy, n. 1, 2001, p. 53.

10. Guillory, John, The Problem of Literary Canon Formation, Chicago-London, The University of Chicago Press, 1993, p. 30. 
docenti. Per elaborare il questionario è risultato indispensabile ricostruire preliminarmente i termini del dibattito sul canone italiano, verificando come quest'ultimo si rapporta a un canone europeo, transnazionale e mondiale; particolarmente utile è stato il confronto con ricerche simili condotte in ambiti diversi dagli studi italiani.

Nello stabilire i criteri di selezione proposti nel questionario, ha svolto un ruolo decisivo il concetto di "common reader" teorizzato da Gallagher sulla scia di Kaplan e Rose che, nel loro The Canon and The Common Reader", lo definiscono come l'oscillazione tra i bisogni e i desideri del lettore medio e gli interessi ideologici dell'élite culturale accademica ${ }^{12}$. Gallagher si è soffermata a descrivere la molteplicità di fattori che influenzano la formazione dei canoni pedagogici: criteri materiali (quali la disponibilità di edizioni, traduzioni, ecc.) e occasionali (come, per esempio, la menzione di un'opera a un convegno), ma anche un insieme di fattori estetici ${ }^{13}$. Basti pensare, per esempio, all'importanza dei giudizi espressi dagli studiosi a voce o in forma pubblica, veicolati ormai da molteplici canali d'informazione. Nella selezione messa in atto nel contesto scolastico, inoltre, come segnala Gallagher, intervengono fattori squisitamente didattici, quali l'utilità tematica di un'opera, il suo valore esemplare per un periodo o movimento culturale, la sua stessa intrinseca natura che lo rende di per sé (in)adatto alla circolazione in ambiti scolastici o comunque ne preclude l'uso generalizzato ${ }^{14}$.

Altrettanto decisivi sono inoltre i criteri ideologici ed etici, come la visione del mondo presentata nell'opera, le questioni di razza e gender, l'affermarsi delle letterature postcoloniali e transnazionali, ecc. Si può aggiungere che gli studi sul canone hanno preso in considerazione anche la relazione tra la formazione del canone e la produzione culturale. Secondo Rakefet Sela-Sheffy, in ogni processo di cambiamento culturale il canone dovrebbe essere inteso piuttosto come un meccanismo di oggettivazione e di naturalizzazione ${ }^{15}$. Lo si potrebbe definire in altri termini come un "ammortizzatore" per controbilanciare un'ac-

11. Kaplan, Carey, Rose, Ellen Cronan, The Canon and the Common Reader, Knoxville, University of Tennessee Press, 1990.

12. Kaplan e Rose sono citati da Gallagher, Susan V., "Contingencies and Intersections", cit., p. 63.

13. Ibid., p. 57.

14. Ibid., pp. 62-63.

15. Sela-ShefFy, Rakefet, "Canon Formation Revisited: Canon and Cultural Production”, Neohelicon, n. 2, 2002, p. 146. 
celerazione del cambiamento ${ }^{16}$. Ciò significa che essendo ciascuno, in qualunque posizione sociale, un agente sia attivo che passivo di questo meccanismo, non basta accusare certi gruppi culturali; bisognerebbe invece rendersi conto del fatto che non tutte le pratiche culturali si rapportano nello stesso modo ai meccanismi di naturalizzazione e affermazione di un valore canonico, che SelaSheffy esprime con il concetto di "esemplare" 17 .

In conclusione, i fattori ricordati da Gallagher costituiscono dei possibili criteri di selezione per i docenti nel momento in cui stilano il programma di letteratura; inoltre un tale ragionamento mette a nudo l'infondatezza dell'esistenza di un canone fisso, precostituito, basato su valori assoluti. Come sottolinea la studiosa, ciò permette di individuare nella pratica quotidiana di scelta e selezione, operata dalle istituzioni scolastiche e dal personale insegnante, non solo un semplice e continuo aggiustamento del canone pedagogico ma un processo capace di determinare l'inserimento di testi considerati non canonici nel canone immaginario ${ }^{18}$. Sebbene il canone possa implicare un'ipotesi intuitiva di "esclusione" di alcuni autori, nella pratica, secondo Guillory, si verifica il contrario, cioè l'inserimento di autori non canonici nel sillabo. Grazie a questa dinamica di esclusione e inclusione, anche gli autori non canonici vengono ammessi de facto nel canone ${ }^{19}$.

In questa prospettiva, la riflessione di Gallagher, prendendo le mosse da Guillory, ci spinge a considerare il peso decisivo delle istituzioni scolastiche nella trasmissione del canone ${ }^{20}$ e a riflettere anche sul ruolo specifico dei docenti nella costituzione del canone pedagogico della letteratura italiana all'estero.

Anche la ricerca condotta da Bernth Lindfors in Sudafrica nel 1992, due anni dopo la fine dell'Apartheid, è stata fonte d'ispirazione per il nostro metodo di indagine. Lindfors ha raccolto 139 descrizioni di corsi in 22 università sudafricane con l'obiettivo di scoprire quali autori africani e quali loro opere fossero lettura obbligatoria nei corsi di letteratura inglese. I dati numerici sono poi stati confrontati con quelli raccolti sei anni prima in 26 università di altri 14 paesi africani dove l'inglese è lingua veicolare, per verificare in che misura il corso di laurea d'inglese fosse stato decolonizzato dopo l'indipendenza ${ }^{21}$.

16. Ibid., p. 150.

17. Ibid., p. 151.

18. Gallagher, Susan V., “Contingencies and Intersections”, cit., p. 57.

19. Guillory, John, The Problem of Literary Canon Formation, cit., p. 1.

20. Gallagher, Susan V., "Contingencies and Intersections", cit., p. 55.

21. Lindfors, Bernth, "African literature teaching in South African university English Departments", Alternation, n. 3, 1996, pp. 5-6. 


\section{IL DIBATTITO SUL CANONE IN ITALIA}

L'indagine empirica di cui questo saggio dà conto si colloca dunque nel contesto del dibattito critico sul canone in generale e sul caso specifico italiano. Anche in Italia studiosi e critici di letteratura si sono confrontati sulla definizione di un canone italiano, ricordiamo almeno il numero speciale di Allegoria del 1998 e, l'anno dopo, Quaderns d'Italia ${ }^{22}$. Non intendiamo qui riassumere le molteplici opinioni e linee di pensiero sviluppatesi sul tema, quanto piuttosto individuare alcuni punti nodali della discussione, utili per fare un confronto con i dati del nostro questionario.

Se è vero che si registra a livello mondiale una crisi dello status della letteratura, tale fenomeno nell'ambito italiano sembra coniugarsi con la messa in discussione del concetto di identità nazionale e, di riflesso, pone la questione del canone inteso come eredità culturale da trasmettere alle nuove generazioni. Anche per Romano Luperini la difficoltà di definizione del canone novecentesco si ricollega alla "progressiva difficoltà della società italiana, nel corso del Novecento, di darsi un'identità culturale e forse un'identità tout court" ${ }^{\prime 2}$. Gli scontri su questo fronte appaiono in modo particolare quando si affronta la questione del rapporto fra tradizione e modernità, che si traduce in una riflessione epistemologica sulla letteratura nel Novecento e, successivamente, sulla necessità di determinare un canone novecentesco, in particolare per la prosa del secondo Novecento ${ }^{24}$. A rinfocolare la discussione contribuisce anche il dualismo fra la letteratura nazionale e dialettale, considerata la forte ripresa del plurilinguismo in ambito letterario ${ }^{25}$.

Un altro punto di discussione è il rapporto tra la letteratura postcoloniale italiana e il canone, al quale Anna Finozzi ha dedicato la tesi di Master coniugando il dibattito sul canone letterario a una ricerca empirica a due facce: la descrizione di una serie di corsi, in atenei italiani ed esteri, che prevedono lo

22. Antonelli, Roberto, Ceserani, Remo, Coletti, Vittorio, Di Girolamo, Costanzo, Ferroni, Giulio, Luperini, Romano, Spinazzola, Vittorio, Caesar, Michael, "Riflessioni sul canone della letteratura italiana nella prospettiva dell'insegnamento all'estero", Quaderns d'Italia, n. 4/5, 1999/2000, pp. 11-46.

23. Luperini, Romano, "Il canone del Novecento e le istituzioni educative", in Merola, Nicola (a cura di), Il canone letterario del Novecento italiano, Milano, Rubbettino, 2000 , p. 17.

24. Ibid., pp. 18-19.

25. Cfr. Sulis, Gigliola, "Ridefinire il canone: i dialettali e le antologie poetiche del Novecento", The Italianist, n. 24, 2004, pp. 77-106. 
studio di scrittori postcoloniali italiani; e le pratiche di "cultural agency" 26 di un autore postcoloniale in particolare, Igiaba Scego ${ }^{27}$. Da questi dati emerge, da una parte, che il postcolonialismo è considerato una disciplina al di fuori delle lettere italiane, come del resto confermato nel manifesto "The Italian Postcolonial" di Cristina Lombardi-Diop e Caterina Romeo: catalogando i testi postcoloniali italiani in quanto "letteratura straniera", il loro insegnamento è ritenuto idoneo solo per i corsi di letterature comparate ${ }^{28}$. Dall'altra parte risulta che il concetto di "trans-nazionalità" potrebbe essere un modo per allargare i confini del canone della letteratura italiana, se essa venisse intesa, come propone Emma Bond, come una caratteristica distintiva dell'Italia, area di migrazione "inbetween" - come esprime il trattino - penisola periferica e territorio particolarmente atto a ospitare scambi e soggettività ibride ${ }^{29}$.

A differenza di quanto sostiene Guillory, gli studiosi italiani che dibattono di canone concordano nell'individuare una componente normativa e restrittiva: l'esclusione e la selezione si basano su giudizi di valore ${ }^{30}$. Per Alfonso Berardinelli, per esempio, "il canone guarda al passato secondo l'ottica della conservazione, ma anche al futuro secondo l'ottica della vitalità permanente e dell'innovazione. La sua chiave è insieme normativa e polemica: scegliere un valore vuol dire indicare un disvalore" ${ }^{31}$. In modo simile anche per Luperini il

26. Il concetto di "cultural agency" viene elaborato da Sandra Ponzanesi la quale sostiene che, facendo parte di uno scambio globale nella sua qualità di "commodified exotica", l'autore postcoloniale diventa egli stesso un agente culturale e così svolge un ruolo attivo nella costruzione del canone: Ponzanesi, Sandra, "Boutique Postcolonialism: Literary Awards, Cultural Value and the Canon", in EAD. The Postcolonial Cultural Industry, Basingstoke, Palgrave Macmillan, 2014, pp. 107-34.

27. FInOzZI, Anna, Trans-nationality and Postcolonialism Through Education and Cultural Agency: Revisiting the Canon of Italian Literature, RMA Thesis Comparative Literary Studies, Utrecht, Utrecht University, 15 agosto 2016, relatore Monica Jansen. Le descrizioni dei corsi in parte sono state raccolte grazie ai contatti presi al convegno "Italia fuori Italia", svoltosi all'Université Paris Nanterre, 2-4 giugno 2016.

28. Lombardi-Diop, Cristina, Romeo, Caterina, "The Italian Postcolonial: A Manifesto", Italian Studies, n. 69, 2014, p. 433.

29. Bond, Emma, "Towards a Trans-national Turn in Italian Studies", Italian Studies, n. 69, 2014, pp. 415-416; si veda anche FInOzZI, Anna, Trans-nationality and Postcolonialism, cit., p. 9.

30. LuPERINI, Romano, "Il canone del Novecento e le istituzioni educative", cit., pp. 20-21; Berardineldi, Alfonso, "Alla ricerca di un canone novecentesco", in Merola, Nicola (a cura di), Il canone letterario del Novecento italiano, cit., pp. 93-103 (p. 94). Si veda anche l'articolo di Olivieri, Ugo M., "I sommersi e i salvati, Appunti su canone e teoria letteraria", s.a., http://www.griseldaonline.it/didattica/sommersi-e-salvati-canonee-teoria-letteraria-olivieri.html

31. Berardinelli, Alfonso, "Alla ricerca di un canone novecentesco", cit., p. 94. 
canone italiano "indica la tavola dei valori prevalente. Essa si traduce poi nell'elenco di libri di cui si prescrive la lettura nell'ambito delle istituzioni educative di una determinata comunità" ${ }^{\prime 2}$. È lo stesso Luperini tuttavia a riconoscere, nella selezione delle opere canoniche, il rapporto dialettico fra le istituzioni letterarie e la scuola, assegnando alla seconda la libertà di "ridefinire incessantemente il canone" 33 , senza che questo impedisca agli organi competenti di tracciare le linee generali e di formalizzare un canone scolastico, seppur aperto ed elastico, in quanto si può contestare e modificare un canone soltanto quando lo si è normalizzato:

In una situazione di crisi dell'insegnamento delle discipline umanistiche come l'attuale, è necessario che il docente di letteratura riacquisti coscienza della propria funzione storico-antropologica: che è quella di conservare la memoria e dunque anche il canone della propria comunità, di continuo aggiornandolo e modificandolo. [...] La funzione insostituibile dell'insegnamento delle materie umanistiche sta anche in questo: che, rendendo visibile il carattere selettivo della memoria storica, rende esplicito il problema dei valori ${ }^{34}$.

Confrontando il dibattito sul canone della letteratura italiana in Italia e oltrefrontiera, il primo elemento di differenza è che i critici italiani sembrano partire da una situazione di crisi della cultura e delle identità nazionali, mentre dagli studi svolti in ambito statunitense o sudafricano emergono le opportunità di emancipazione e inclusione offerte dal canone. La riflessione dei critici italiani sui meccanismi di selezione è ispirata in primo luogo da criteri estetici, eticoideologici, e meno da condizioni materiali o pragmatiche. C'è tuttavia qualche segno di riorientamento; Luperini segnala per esempio un crescente interesse per la ricezione grazie "allo spostamento complessivo dell'attenzione teorica e critica, che si è andata sempre più concentrando sul lettore invece che sull'autore o sul testo" ${ }^{\prime 3}$. Malgrado ciò, la costruzione del canone italiano sembra motivata dallo stabilire valori pedagogici e morali piuttosto che dalla prassi di insegnamento. L'aspetto pragmatico risulta invece decisivo nella definizione di un canone della letteratura italiana all'estero, in quanto, come segnala Massimo

32. Luperini, Romano, "Il canone del Novecento e le istituzioni educative", cit., p. 11.

33. Ibid., p. 20.

34. Ibid., p. 21.

35. Ibid., p. 11. 
Lucarelli, gli studenti hanno meno tempo dei loro coetanei italiani per studiare i testi letterari ${ }^{36}$.

Nel caso italiano, inoltre, si continua a sentire la predominanza della tradizione storicistica, per cui il canone pedagogico si identifica con lo studio della storia della letteratura e ogni autore viene trattato in quanto esemplare di una corrente o un periodo; come fa notare ironicamente Berardinelli, l'organizzazione dello studio della letteratura in tale chiave continua a esistere nel contesto italiano, a differenza di quello francofono o anglosassone, anche in un'epoca come la nostra che ha proclamato la fine della storia ${ }^{37}$. Eppure fra gli studiosi emerge la tendenza a superare o comunque a contemperare tale approccio facendo studiare le opere come strumento di esplorazione di un contesto, di un mondo nella sua totalità ${ }^{38}$. In questa direzione andava anche un progetto pilota sperimentato in Emilia Romagna negli anni Novanta in cui il curriculum di letteratura, tentando una mediazione fra approccio storicista ed esemplarità dell'opera, era costruito su tipologie di unità didattiche diverse. Il fine era quello di proporre agli studenti una visione della letteratura non tanto come "un patrimonio da trasmettere, quanto un territorio da esplorare: un territorio accidentato, ricco di sorprese, dai confini incerti". Una prospettiva dunque che "vede lo studente non più come destinatario passivo di una conoscenza precostituita da assimilare, ma come il costruttore attivo della propria conoscenza ${ }^{39}$.

Un altro punto ricorrente nel dibattito è la questione del rapporto fra la contemporaneità e il passato. Si avverte la necessità di rileggere gli autori del passato alla luce di valori, visioni e chiavi interpretative del presente; pertanto diventa indispensabile stabilire preliminarmente quali possano essere i valori guida. Ciò implica una rilettura critica del Novecento visto che, come afferma Luperini, non esiste ancora un canone scolastico del Novecento e stenta anche ad affermarsi l'idea che alcuni autori del Novecento possano essere considerati

36. Lucarelli, Massimo, "Il canone della letteratura italiana in Francia nei programmi di Agrégation (1900-2013)", RELIT - Revista de Estudios Literários do NEIITA, n. 3, 2011, p. 76.

37. Berardineldi, Alfonso, "Alla ricerca di un canone novecentesco", cit., p. 94.

38. La proposta di rinnovamento dell'insegnamento della letteratura nella scuola secondaria su base "modulare" è descritta nel volume Colombo, Adriano, Corradini, Simonetta, La letteratura per unità didattica, Firenze, La Nuova Italia, 1996. Il testo di Colombo, intitolato "La letteratura per unità didattiche", è ora disponibile on line su http://www.adrianocolombo.it/edulet/edulet06.pdf

39. Ibid., p. 6. 
dei classici ${ }^{40}$. Tale riflessione appare tanto più congrua quando si pensa agli elementi di maggiore incertezza che caratterizzano i vari canoni dell'italiano nei programmi scolastici italiani e all'estero. In fondo le maggiori differenze si riconoscono proprio nella selezione del canone novecentesco, mentre molto più stabili, nonostante certe differenze fra realtà italiana ed estera, sono le liste degli autori dei secoli precedenti. Nonostante la difficoltà di "storicizzare il presente" ", l'impresa deve essere compiuta, all'interno del curriculum del triennio, almeno con riguardo al periodo che si estende fino al 1956, ritiene Luperini $^{42}$. D'altronde, lo studioso ricorda che De Sanctis fece tale opera di selezione anche per il suo presente, includendo Leopardi e Manzoni nel canone della letteratura italiana. Inoltre, la conoscenza del Novecento appare essenziale proprio per comprendere epoche diverse dalle nostre e interrogare il passato con l'ottica del presente. Non si può, per Luperini, continuare a leggere Dante con l'ottica del passato, bisogna piuttosto ripensare l'approccio ai classici anche da un punto di vista intertestuale proponendo, per esempio, la lettura di Dante attraverso la poesia novecentesca di Montale ed Eliot. Inoltre lo studioso, interrogandosi sull'insegnabilità dei classici, rivendica la necessità di superare l'approccio cronologico che vedrebbe inesorabilmente il Novecento oggetto di studio solo alla fine di un lungo percorso di storia letteraria, suggerendo piuttosto un curriculum che favorisca l'intersecazione di epoche vicine e lontane, in "reciproca combustione"

Centrale nel dibattito è dunque la questione di come dare corpo al Novecento, quali autori includere, quale datazione adottare. Sembra prevalere un disordine novecentesco nei confronti dell'ordine della tradizione, il che offre anche delle libertà di scelta. Tuttavia, secondo Olivieri, dal momento che nel Novecento gli istituti letterari (i generi, gli stili, i registri linguistici e tematici) e le istituzioni letterarie tradizionali (editoria, insegnamento, critica) sembrano "funzionare meno come base di una gerarchia di valori letterari", diventa importante avere "un modello di descrizione teorica del testo per potere attuare lo scarto che è sempre necessario per stabilire un canone". Per Olivieri il canone "può usare la sua 'inattualità' come misura e reinterpretazione della tradizione

40. Luperini, Romano, "Il canone del Novecento e le istituzioni educative", cit., p. 16.

41. Ibid., p. 16.

42. Ibid.

43. Ibid. 
dal punto di vista del contemporaneo" ${ }^{\text {"4 }}$. Per fare ciò diventa urgente discutere sul Novecento, riflettendo in chiave epistemologica sul valore dello studio della letteratura della contemporaneità. Peraltro, l'esigenza di includere nel canone testi della tradizione che sappiano dialogare con la contemporaneità rafforza anche l'importanza del criterio etico-testimoniale della selezione.

In conclusione, dagli studi consultati sembra emergere in Italia un approccio istituzionale al tema del canone pedagogico, mentre Gallagher e Guillory partivano piuttosto dall'esperienza dei docenti. Si noti tuttavia che Luperini segnala il ruolo attivo dei docenti italiani nella selezione degli autori quando ribadisce la libertà della scuola di "ridefinire incessantemente il canone", senza che questo impedisca agli organi competenti di tracciare linee generali e formalizzare un canone scolastico ${ }^{45}$.

Un breve cenno merita la situazione francese. Un'analisi condotta da Lucarelli evidenzia come siano i concorsi per l'insegnamento nelle scuole secondarie, Agrégation e CAPES, a informare le scelte degli autori proposti all'università. Inoltre, il confronto fra gli argomenti dell'Agrégation e gli autori presenti nelle liste ministeriali del triennio italiano (Brocca 1995) permette a Lucarelli di concludere che il 70\% degli autori della lista Brocca coincide con quelli selezionati per l' $A$ grégation. Lucarelli nota infine, da un lato, un maggiore interesse per il Rinascimento come periodo letterario e culturale, dall'altro, una presenza limitata della letteratura contemporanea del secondo Novecento: 14 su 57 argomenti, comunque maggiore rispetto ai programmi italiani, anche se Calvino risulta l'unico vero autore "canonico" del secondo Novecento, sia in Italia che in Francia ${ }^{46}$.

\section{RisuLTATI DI UN'INCHIESTA EMPIRICA NEGLI ATENEI BELGI E OLANDESI}

Tracciato il quadro teorico, l'obiettivo del presente studio è duplice: da un lato elaborare uno strumento di rilevamento con il quale analizzare il canone pedagogico dei programmi di letteratura italiana nelle università olandesi e belghe e, in un'ulteriore fase del lavoro, in altre università estere; dall'altro, raccogliere dati sulla scelta degli autori, sui criteri di scelta e sulla posizione dei docenti

44. Olivieri, Ugo M., "I sommersi e i salvati, Appunti su canone e teoria letteraria", cit., s.p. (per le tre citazioni).

45. Luperini, Romano, "Il canone del Novecento e le istituzioni educative", cit., p. 21.

46. Lucarelli, Massimo, "Il canone della letteratura italiana in Francia nei programmi di Agrégation”, cit., pp. 79-80. 
nei confronti delle riforme e dei cambiamenti in corso. A tale scopo sono state formulate le seguenti domande:

1. Quali autori sono trattati nei corsi di letteratura italiana (non panoramici) nelle accademie olandesi e belghe, e con quale frequenza?

2. Quali criteri o motivazioni hanno maggiormente determinato la selezione degli autori?

2.1 La frequenza del criterio "autore canonico" varia secondo il livello di insegnamento (triennale o specialistica)?

2.2 La frequenza del criterio "attrazione per il common reader" varia secondo il livello di insegnamento?

3. Quali fattori hanno determinato cambiamenti nella selezione degli autori?

4. Quali autori tra Dante, Petrarca, Ariosto, Leopardi, Manzoni, Verga, Pirandello, Pasolini, Morante, Eco, Baricco e Ferrante sono ritenuti imprescindibili?

5. Quali autori e quali generi verrebbero selezionati per un ipotetico corso panoramico sulla letteratura italiana dagli anni Sessanta in poi?

6. Qual è l'opinione dei docenti sul futuro dell'insegnamento della letteratura italiana e sull'eventuale necessità di riformare il detto insegnamento?

Sono pochi gli studi quantitativi dedicati al fenomeno del canone pedagogico. Come si è visto, per raccogliere i dati Lindfors si era basato sulle descrizioni dei corsi e sulle liste delle letture obbligatorie, precisando tuttavia d'aver trascorso in Sudafrica due mesi durante i quali aveva incontrato molti colleghi ${ }^{47}$. Lindfors si limita a un'analisi quantitativa degli autori che figurano sulle liste di letture obbligatorie, del numero di opere per autore e del livello dell'insegnamento. Il presente studio, che invece vuole indagare anche cosa motivi la scelta di un autore, ha necessitato il ricorso sia al questionario che all'intervista, perché l'accesso alle descrizioni dei corsi sui siti delle università è spesso limitato, e di conseguenza, le considerazioni dei docenti non sono direttamente osservabili.

Il rilevamento di dati tramite intervista e questionario permette di esaminare: 1) la scelta degli autori e i criteri di selezione adottati dagli insegnanti 2) le loro motivazioni 3) le loro opinioni circa il futuro dell'insegnamento della letteratura italiana all'estero 4) alcuni dati socio-demografici.

Per ogni partecipante sono stati raccolti dati relativi ad alcuni corsi non panoramici per i quali il docente ritiene di godere di una relativa autonomia di

47. LINDFORS, Bernth, "African literature teaching in South African university English Departments”, cit., pp. 5-14. 
scelta. È stato chiesto ai docenti di fornire alcuni dati generali: titolo del corso, numero di crediti, livello, impostazione, modalità della lista di letture (fissa o variabile) ed eventuale uso di traduzioni. In seguito, i partecipanti hanno dovuto elencare gli autori trattati e le motivazioni della scelta. Riportiamo qui di seguito le possibili risposte alla domanda "Perché ha scelto quest'autore?" (risposte multiple ammesse $)^{48}$.

1. Perché è un autore migrante o postcoloniale.

2. Per questioni di gender.

3. Perché va incontro al gusto degli studenti.

4. Perché è un autore canonico.

5. Perché esprime una certa visione etico-politica del mondo e della società.

6. Per una questione di gusto personale.

7. Perché mi è stato segnalato o perché ne ho sentito parlare a un convegno.

8. Perché il testo è attraente per il lettore comune.

9. Per interessi personali di ricerca.

10. Perché tratta tematiche attinenti all'interesse degli studenti.

11. Perché rientra nelle tematiche affrontate dal corso (p. es. Celati in un corso sul paesaggio nella letteratura).

12. Per il valore estetico del testo.

13. Perché esiste la traduzione in olandese.

14. Altro.

Una seconda parte del questionario conteneva le tre domande seguenti:

1. Ha mai modificato l'elenco degli autori trattati in un corso in seguito a 1) riforme del programma, 2) valutazioni degli studenti, 3) nuovi interessi e progetti di ricerca in corso, 4) presenza di studenti provenienti da altri indirizzi o corsi di laurea, 5) scelte e motivi didattici, 6) proposte degli studenti, 7) altri motivi.

2. Se Le venisse assegnato un nuovo corso panoramico sulla letteratura italiana (con un'impostazione a scelta) dagli anni Sessanta in poi, inserirebbe 1) autori finora non ancora presenti nelle antologie o storie della letteratura, 2) autori degli anni Duemila, 3) autori in dialetto, 4) testi di genere, 5) testi poetici,

48. Si vedano Guillory, John, Cultural Capital, cit.; Verboord, Marc, "Veranderingen in benaderingen van het literatuuronderwijs. Literatuuropvattingen overgedragen door docenten Nederlands", in Dorleijn, Gillis, van Rens, Kees (a cura di), De productie van literatuur. Het literaire veld in Nederland 1800-2000, Nijmegen, Vantilt, 2006. Quest'ultimo saggio è dedicato all'evoluzione dell'insegnamento della letteratura neerlandese nella scuola superiore olandese e alle idee elaborate a riguardo dai docenti. 
6) testi saggistici, 7) bestseller, 8) autori non canonici, 9) autori postcoloniali, 10) autori migranti, 11) autori italo-ebrei?

3. Gli studenti d'italiano si possono laureare senza aver studiato 1) Dante, 2) Petrarca, 3) Ariosto, 4) Leopardi, 5) Manzoni, 6) Verga, 7) Pirandello, 8) Pasolini, 9) Morante, 10) Eco, 11) Baricco, 12) Ferrante? ${ }^{49}$.

Altre due domande a risposta aperta erano volte a indagare le aspettative riguardo al futuro dell'insegnamento della letteratura italiana nelle accademie estere.

La parte conclusiva del questionario ha raccolto informazioni circa le caratteristiche anagrafiche e la carriera scolastica e universitaria dei partecipanti, con la finalità di poter operare - in un'ulteriore fase della ricerca che includa altre accademie estere e, di conseguenza, un numero più elevato di partecipanti - un confronto tra gli elementi distintivi dei rispondenti (p. es. diploma/laurea/dottorato conseguiti in Italia o all'estero ${ }^{50}$.

Per la prima fase di questa ricerca sono state prese in esame le università olandesi e belghe che offrono corsi di letteratura italiana e sono stati invitati a compilare il questionario i docenti di letteratura italiana ${ }^{51}$. Si consideri che in gran parte delle accademie belghe vi è una sola cattedra di letteratura italiana, il che spiega il numero limitato di interviste; i dati in questa sezione fanno riferimento a dieci rispondenti, sei dei quali in attività presso accademie olandesi e quattro presso quelle belghe. Il campione include sei partecipanti di nazionalità olandese o belga e quattro di nazionalità italiana, i quali hanno conseguito sia il diploma che la laurea e il dottorato in Italia.

Data la struttura complessa del questionario, lo abbiamo presentato sotto forma di intervista ${ }^{52}$. A partire dalle risposte sono state costituite due banche

49. Nella lista di autori proposti abbiamo voluto inserire sia autori classici come Dante, Petrarca e Manzoni, sia autori degli anni Duemila la cui opera conosce un'ampia diffusione dentro e fuori dagli atenei. cit.

50. VERBOORD, Marc, "Veranderingen in benaderingen van het literatuuronderwijs",

51. Le università olandesi che hanno partecipato al questionario sono le seguenti: Rijksuniversiteit Groningen, Universiteit van Amsterdam, Universiteit Leiden, Universiteit Utrecht. Le università belghe che hanno aderito sono: Universiteit Gent, KU Leuven, Vrije Universiteit Brussel, Université Libre de Bruxelles, Université de Liège, Université de Namur. Non sono state incluse le università che non offrono corsi monografici di letteratura italiana nei loro piani di studio (esempio, le lauree che preparano alla traduzione e all'interpretariato).

52. FIELD, Andy, Discovering Statistics using IBM SPSS Statistics, Los Angeles, Sage, 2014. Le analisi di seguito svolte sono state realizzate con il pacchetto statistico SPSS. 
dati: una, ideata per contenere le informazioni sui rispondenti, l'altra, per accogliere le loro motivazioni ${ }^{53}$.

Passiamo ai risultati.

1. In totale, la banca dati sugli autori presenti nei curricula comprende 117 menzioni, di cui 71 riguardano autori scelti da due o più docenti e 46 da un solo docente. Dante, per esempio, è proposto da cinque docenti, mentre Ungaretti da un solo. La Tabella 1 mostra la percentuale per ogni autore selezionato da due o più docenti, e presenta quindi un quadro degli autori più ricorrenti nei curricula olandesi e belgi.

Tabella 1. Percentuale di scelte per autore

\begin{tabular}{|l|l|}
\hline$\%$ & Autore \\
\hline 50 & Dante, Svevo, Calvino \\
\hline 40 & Verga, Pirandello, Fenoglio \\
\hline 30 & Petrarca, Boccaccio, Ariosto, Leopardi, Pavese, Pasolini, Sciascia, Saviano \\
\hline 20 & $\begin{array}{l}\text { Machiavelli, D’Annunzio, Lussu, Gadda, Primo Levi, Tomasi di Lampedusa, } \\
\text { Tabucchi, Camilleri }\end{array}$ \\
\hline
\end{tabular}

Gli autori più citati sono (in ordine cronologico): Dante, Svevo e Calvino (50\% dei rispondenti). Il 40\% dei docenti tratta Verga, Pirandello, Fenoglio. Il $30 \%$ fa studiare Petrarca, Boccaccio, Ariosto, Leopardi, Pavese, Pasolini, Sciascia, Saviano. Un quinto tratta Machiavelli, D’Annunzio, Lussu, Gadda, Primo Levi, Tomasi di Lampedusa, Tabucchi, Camilleri.

2. Ai docenti che hanno partecipato all'indagine si è chiesto quali siano stati i criteri che hanno condotto alla scelta di un autore. La Tabella 2 mostra la frequenza dei criteri per i 117 autori selezionati.

53. Per la prima fase dell'indagine, che si limita alle accademie olandesi e belghe, è stata applicata una procedura statistica di tipo descrittivo che prevede la valutazione di frequenž (e percentuali di risposta) e di associazioni mediante Chi quadrato. Per la determinazione della significatività statistica dei risultati è stato considerato un livello di $\mathrm{p}<0.05$. 
Tabella 2. Percentuale di docenti per criterio di selezione

\begin{tabular}{|l|l|}
\hline$\%$ & Motivazione \\
\hline 79 & perché è un autore canonico \\
\hline 79 & perché esprime una certa visione etico-politica del mondo e della società \\
\hline 76 & $\begin{array}{l}\text { perché rientra nelle tematiche affrontate dal corso (p. es. Celati in un corso sul } \\
\text { paesaggio nella letteratura) }\end{array}$ \\
\hline 74 & per il valore estetico del testo \\
\hline 54 & per una questione di gusto personale \\
\hline 50 & perché tratta tematiche attinenti allinteresse degli studenti \\
\hline 46 & per interessi personali di ricerca \\
\hline 25 & perché il testo potrebbe piacere al lettore comune \\
\hline 23 & perché esiste la traduzione in olandese/ inglese \\
\hline 20 & perché va incontro al gusto degli studenti \\
\hline 13 & per questioni di gender \\
\hline 13 & Altro \\
\hline 5 & perché è un autore migrante/postcoloniale \\
\hline 0 & perché mi è stato segnalato o perché ne ho sentito parlare a un convegno \\
\hline
\end{tabular}

I criteri maggiormente adottati sono: il carattere canonico, la visione eticopolitica, la pertinenza rispetto alle tematiche del corso, il valore estetico dell'opera, il gusto personale, l'attinenza a tematiche che interessano gli studenti o agli ambiti di ricerca del docente ${ }^{54}$. Pur essendo la canonicità il criterio più ricorrente, il criterio "perché esprime una certa visione etico-politica del mondo e della società", altrettanto frequente, testimonia l'interesse per approcci di tipo contestuale. Mentre Gallagher ritiene che i convegni e gli incontri casuali siano fattori che influiscono sulla costruzione dei sillabi ${ }^{55}$, nel nostro studio tale criterio non è stato indicato da nessun partecipante. Dai dati riportati nella Tabella 2 , inoltre, sembra risultare che i docenti interrogati tengano poco conto dei gusti degli studenti e di quelli del lettore comune. La disponibilità di testi in traduzione (in olandese o in inglese), infine, conta soltanto nei corsi che non sono impartiti in italiano.

54. Altri criteri sono stati selezionati meno frequentemente. Per esempio, un docente ha selezionato autori poco studiati e poco noti perché costituiscono una novità per lo studente e un arricchimento per il programma. Un altro docente ha selezionato degli autori perché esemplificano bene alcuni aspetti teorici.

55. Gallagher, Susan V., "Contingencies and Intersections”, cit., p. 59. 
2.1 La Tabella 3 mostra la frequenza con cui è stato selezionato il criterio della canonicità secondo il livello di insegnamento (laurea triennale e specialistica).

Tabella 3. Frequenza del criterio "autore canonico"

\begin{tabular}{|l|l|l|l|l|}
\hline \multicolumn{2}{|l|}{ Perché ha scelto l'autore? } & Triennale & specialistica & Totale \\
\hline \multirow{2}{*}{ Perché è un autore canonico? } & no & $17(19,3 \%)$ & $8(27,6 \%)$ & $25(21,4 \%)$ \\
\cline { 2 - 6 } & si & $71(80,7 \%)$ & $21(72,4 \%)$ & $92(78,6 \%)$ \\
\hline
\end{tabular}

Dai dati risulta che l' $80,7 \%$ degli autori trattati nei corsi della triennale sono stati scelti perché ritenuti canonici, contro il $72,4 \%$ nei corsi di specialistica. Tale differenza è risultata non significativa al test del Chi quadrato ${ }^{56}$, in altri termini, la percentuale di autori selezionati perché ritenuti canonici non varia tra laurea triennale e specialistica. Gallagher ritiene che il canone immaginario svolga un ruolo importante nei corsi di laurea il cui obiettivo sia trattare testi o autori frequentemente citati o discussi dalla critica accademica ${ }^{57}$. I risultati del nostro studio lo confermano e indicano inoltre che, per il nostro campione, il canone immaginario costituisce un importante punto di riferimento in ogni ciclo di studi.

$2.2 \mathrm{Si}$ è valutata poi l'associazione tra il criterio "attrazione per il common rea$d e r$ " e il livello di insegnamento.

Tabella 4. Common reader e livello di insegnamento

\begin{tabular}{|l|l|l|l|l|}
\hline \multicolumn{2}{|l|}{ Perché ha scelto l'autore? } & Triennale & Specialistica & Totale \\
\hline \multirow{2}{*}{$\begin{array}{l}\text { Perché il testo è attraente per il lettore } \\
\text { comune? }\end{array}$} & no & $66(75,0 \%)$ & $22(75,9 \%)$ & $88(75,2 \%)$ \\
\cline { 2 - 6 } & sì & $22(25,0 \%)$ & $7(24,1 \%)$ & $29(24,8 \%)$ \\
\hline
\end{tabular}

Il criterio riguarda il 25\% degli autori studiati nella triennale, contro il $24,1 \%$ nella specialistica. Neppure tale differenza è risultata significativa al test del Chi

56. $C h i^{2}=.887, d f=1, p=.346$.

57. Gallagher, Susan V., "Contingencies and Intersections”, cit., p. 66. 
quadrato $^{58}$, ossia l'attrattività per il lettore comune non cambia influenza secondo il livello di insegnamento.

3. La terza domanda riguarda le motivazioni che spingono i docenti a cambiare autore.

Tabella 5: Percentuale di docenti per motivazione

\begin{tabular}{|l|l|}
\hline$\%$ & Fattori che hanno contribuito al cambiamento \\
\hline 100 & interessi e progetti di ricerca in corso \\
\hline 78 & scelte e motivi didattici \\
\hline 67 & valutazione degli studenti \\
\hline 56 & riforme del programma \\
\hline 44 & presenza di studenti provenienti da altri indirizzi/corsi di laurea \\
\hline 11 & proposte degli studenti \\
\hline 11 & Altro \\
\hline
\end{tabular}

La Tabella 5 mostra che i dieci docenti del campione hanno dichiarato tutti di aver scelto gli autori in programma in funzione dei propri interessi e progetti di ricerca. Si noti che comunque anche motivi didattici hanno influito, e così le valutazioni degli studenti. Un ultimo fattore importante è rappresentato dalle riforme dei programmi. Oltre a evidenziare che la selezione degli autori è in costante evoluzione ${ }^{59}$, le risposte sembrano suggerire che il docente tiene comunque conto dello studente per stabilire i contenuti dei propri corsi. Il canone pedagogico si presenta quindi come un concetto tipicamente dinamico.

4. La Tabella 6 riguarda gli autori ritenuti imprescindibili, tra Dante, Petrarca, Ariosto, Leopardi, Manzoni, Verga, Pirandello, Pasolini, Morante, Eco, Baricco e Ferrante.

Tabella 6: Percentuale di docenti per autore proposto come imprescindibile

\begin{tabular}{|l|l|}
\hline$\%$ & Autori ritenuti imprescindibili \\
\hline 80 & Dante, Manzoni, Pirandello, Pasolini, Eco \\
\hline
\end{tabular}

58. $C h i^{2}=.009, d f=1, p=.926$.

59. Cfr. Antonelli, Roberto et alii, "Riflessioni sul canone della letteratura italiana", cit., p. 21. 


\begin{tabular}{|l|l|}
\hline 70 & Petrarca, Leopardi, Verga \\
\hline 40 & Ariosto \\
\hline 30 & Morante, Baricco, Ferrante \\
\hline
\end{tabular}

Gli autori meno imprescindibili risultano essere Morante, Ferrante e Baricco, scelti solo dal 30 per cento dei docenti. Dante, Manzoni, Pirandello, Pasolini, Eco, Petrarca, Leopardi e Verga riscuotono il maggiore consenso. Colpisce che tra gli autori più contrassegnati figurino anche due contemporanei, Pasolini ed Eco, mentre, se si guarda agli autori proposti nei piani di studio, Eco viene citato una sola volta. Comunque, l'inclusione nel canone pedagogico di autori sia classici che contemporanei evidenzia che entrambe le categorie sono considerate "insegnabili" ${ }^{0}$, nonostante non siano sempre di facile lettura.

5. Nella Tabella 7 sono riportate le risposte alla quinta domanda, ossia autori e generi che verrebbero scelti per un ipotetico corso panoramico sulla letteratura italiana dagli anni Sessanta in poi.

\section{Tabella 7: Percentuale di docenti per tipo di autori e generi}

\begin{tabular}{|l|l|}
\hline$\%$ & Tipo di autori e generi \\
\hline 90 & testi di genere, testi poetici, testi saggistici, bestseller, autori non canonici \\
\hline 80 & autori degli anni Duemila, autori postcoloniali \\
\hline 70 & $\begin{array}{l}\text { autori migranti, autori finora non ancora presenti nelle antologie o storie della } \\
\text { letteratura }\end{array}$ \\
\hline 50 & autori ebrei \\
\hline 40 & autori in dialetto \\
\hline
\end{tabular}

Dai dati risulta che solo il 40 per cento dei partecipanti è disponibile a includere autori in dialetto e solo la metà prenderebbe in considerazione autori italoebrei. Le altre proposte elencate qui sopra sono accolte in modo più positivo (dal 90 al 70\%). Questa selezione potenziale appare quindi abbastanza "inclusiva" se paragonata al canone pedagogico come sopra delineato; testimonia infatti una maggiore apertura agli autori non canonici. Dall'insieme delle risposte si ricava che, almeno nelle intenzioni, i docenti non tendono né a esclu-

60. Gallagher, Susan V., "Contingencies and Intersections", cit., p. 66.

61. Guillory, John, Cultural Capital, cit., p. 7. 
dere il non canonico né a istituzionalizzare il canonico, rendendo definitivi i propri programmi.

6. L'ultima domanda riguarda l'opinione dei partecipanti riguardo al futuro dell'insegnamento della letteratura italiana e all'eventuale necessità di riformarlo. In Olanda e nella parte neerlandofona del Belgio i docenti si sono mostrati piuttosto pessimisti e preoccupati anzitutto per il calo delle iscrizioni. Secondo alcuni di loro l'interesse degli studenti sembra più di prima condizionato da scelte professionali a breve termine. A questa tendenza i docenti sembrano reagire proponendo programmi in cui allo studio approfondito di autori canonici si sostituiscono programmi più liberi e variegati; inoltre, almeno in Olanda, si dimostrano aperti ad approcci di insegnamento interdisciplinari. In entrambi i paesi, i docenti sembrano disposti a esplorare anche la "traducibilità" 62 dei testi selezionati, andando oltre i limiti dell'opera scritta e creando spazio per l'analisi di adattamenti cinematografici e altri.

\section{ConCLusioni}

I risultati emersi dalla ricerca empirica qui presentata, anche se limitati agli atenei belgi e olandesi, suscitano alcune riflessioni di carattere generale sul ruolo che il canone pedagogico all'estero svolge nel processo di selezione e valorizzazione del patrimonio letterario italiano, da sempre considerato uno degli emblemi dell'identità nazionale.

Nonostante dal dibattito sul canone in Italia emergano segnali di cambiamento e si avverta l'esigenza di sganciarsi da un approccio storicista al fine di rivalutare lo studio della letteratura alla luce di istanze estetiche ed etiche contemporanee, è evidente che ogni tentativo di modifica deve fare i conti con il timore di intaccare uno degli elementi considerati fondanti dell'identità italiana. Diversamente dalla situazione italiana, il canone pedagogico all'estero appare il risultato di un approccio più pragmatico che ideologico, anche per far fronte a circostanze economico-sociali sfavorevoli per le discipline umanistiche, oltre a quelle legate all'insegnamento della lingua. Da un lato, i docenti all'estero continuano a insegnare i classici della letteratura italiana, dall'altro, però, si interrogano criticamente, e forse più liberamente, su quali autori proporre e quali escludere, arrivando ad operare delle scelte talvolta anche diverse rispetto ai colleghi in Italia. A causa delle difficoltà contestuali, lontani dalla comunità che

62. Cfr. Antoneldi, Roberto, et alii, "Riflessioni sul canone della letteratura italiana", cit., pp. 19-23. 
si identifica almeno idealmente con il canone letterario, i docenti all'estero avvertono ancora più fortemente l'esigenza di dimostrare il valore della letteratura italiana privilegiando quelle opere in grado di stabilire un dialogo con il presente. In questo senso i docenti si dimostrano consapevoli della loro funzione di mediatori culturali fra due mondi e due culture vive e dinamiche (quella italiana e quella del Paese in cui si insegna). Tale tendenza si traduce in un canone tendenzialmente inclusivo, aperto alla molteplicità e varietà delle espressioni letterarie anche contemporanee, non escludendo un certo grado di arbitrarietà. Così facendo, i docenti, oltre che influire sulla formazione del canone immaginario, preservano la visibilità della letteratura italiana nel mercato globale delle lettere. Confrontando infine il risultato "inclusivo" della domanda 2 con la risposta prevalentemente "esclusiva" alla domanda 3, si potrebbe concludere che il patrimonio culturale italiano viene inteso in termini "monumentalizzanti" quando gli interrogati sono spinti a pensare ai "classici" da selezionare, e in termini "democratici" quando si chiede loro di riflettere sul valore contemporaneo delle opere da insegnare.

La correlazione fra canone pedagogico e canone immaginario sembra inoltre rafforzata dal criterio di selezione più frequente: gli interessi personali di ricerca. Dal momento che tali interessi si spingono spesso verso la contemporaneità e includono autori ancora ai margini dei programmi (e della ricerca) in Italia, i docenti non si limitano a influenzare e modificare il canone pedagogico ma, costruendo un apparato critico, pongono le basi per accogliere questi autori nel canone italiano del futuro, come succede per esempio grazie ai volumi che risultano da convegni sulla letteratura italiana contemporanea. In questa prospettiva, il concetto di canone immaginario come deposito di memorie nazionali è in realtà il prodotto di un processo di elaborazione e selezione che avviene in parte anche al di là dei confini nazionali, fortemente debitore dei processi di ricezione (e traduzione all'estero). La periferia rispetto ai centri di elaborazione del canone letterario in patria (e la conseguente libertà d'azione) potrebbe così rivelarsi uno spazio ideale per trasformare il canone italiano a partire da una prospettiva transnazionale.

Anche dal punto di vista didattico i risultati emersi dal questionario invitano a una riflessione di carattere generale. Nel caso dell'Olanda, la scelta di proporre lo studio della letteratura italiana in rapporto con altri media, pur essendo in parte la risposta a esigenze concrete, potrebbe implicare anche profondi mutamenti nel canone pedagogico, non solo favorendo un confronto diretto fra opere letterarie e altre opere artistiche, ma soprattutto privilegiando gli autori 
che sono stati "tradotti" nel senso più ampio del termine e che si prestano dunque a essere studiati grazie alla loro transmedialità.

I segnali che sembrano emergere dall'indagine qui presentata sono dunque aperti a più interpretazioni: confermano la dinamicità del canone pedagogico e il ruolo decisivo dei docenti nella creazione di un canone immaginario più inclusivo, ma pongono anche degli interrogativi sulla ricezione del canone letterario italiano all'estero.

\author{
Natalie Dupré \\ KU Leuven \\ Monica JANSEN \\ Universiteit Utrecht \\ Inge LANSLOTS \\ KU Leuven \\ Maria Bonaria URBAN
} Universiteit van Amsterdam 